\title{
Insights into Functional Tetracycline/Antioxidant Containing Chitosan Hydrogels as Potential Bio-Active Restorative Materials: Structure, Function and Antimicrobial Activity
}

\author{
Victoria Tamara Perchyonok ${ }^{1}$, Shengmiao Zhang ${ }^{2}$, Nicki Basson ${ }^{3}$, Sias Grobler ${ }^{3}$, \\ Theunis Oberholzer ${ }^{1}$, Ward Massey ${ }^{1}$ \\ ${ }^{1}$ School of Dentistry and Oral Health, Griffith University, Gold Coast, Australia \\ ${ }^{2}$ School of Material Science and Engineering, East China University of Science and Technology, Shanghai, China \\ ${ }^{3}$ Oral and Dental Research Institute, Faculty of Dentistry, University of the Western Cape, Cape Town, South \\ Africa \\ Email: tamaraperchyonok@gmail.com
}

Received 15 December 2013; revised 20 January 2014; accepted 28 January 2014

Copyright (C) 2014 by authors and Scientific Research Publishing Inc.

This work is licensed under the Creative Commons Attribution International License (CC BY).

http://creativecommons.org/licenses/by/4.0/

CC) (i) Open Access

\section{Abstract}

The human inflammatory periodontal diseases are amongst the most common of chronic diseases to affect adults. Periodontitis is regarded as "an inflammatory lesion, mediated by complex hostparasite interactions, that leads to the loss of connective tissue attachment to root surface cementum and adjacent alveolar bone". Substantial data are available in the literature on the role of reactive oxygen species (ROS) and antioxidants in disorders such as the inflammatory diseases. However, remarkably little information is available on the periodontal diseases, which show many of the pathological features of other chronic inflammatory diseases. The periodontal tissues also provide an ideal medium within which to study mechanisms of ROS mediated tissue damage and of antioxidant defense in response to bacterial colonisation, through the non-invasive collection of GCF. The objectives of this study are to evaluate the novel chitosan based functional drug delivery systems which can be successfully incorporated into "dual action bioactive restorative materials" containing common antibiotics such as tetracycline, krill oil, aloe and aspirin as commonly used antioxidant species. Methods: The novel hydrogels will be investigated with respect to the antioxidant capacity and drug release capacity of the tetracycline from the designer drug delivery system, the use of SEM imaging for the characterization of the surfaces and reactive features of novel materials with antimicrobial potential. Results: A steady slow release of tetracycline, while 
maintaining antibiotic effects against the tested bacteria, for at least 10 days was shown from designer chitosan-antioxidant hydrogels. Within the limitations of the study design chitosan-antioxidant hydrogels are suitable materials for functional restorative and periodontal applications in vitro. The addition of antioxidants to the tetracycline containing prototype delivery system had a beneficial effect on the design of the hydrogel by slowing down the release of tetracycline and thereby enabling a sustainable antifungal activity over time.

\section{Keywords}

\section{Chitosan; Hydrogels; Tetracycline; Reactive Oxygen Species; Antioxidants; Functional Biomaterials}

\section{Introduction}

The human inflammatory periodontal diseases are amongst the most common of chronic diseases to affect adults. In the UK, $69 \%$ of adults have early signs of disease and only 5\% are completely free from clinical signs of inflammation [1]. The periodontal complex comprises alveolar bone, periodontal ligament, root cementum, and the overlying gingival (gum) tissues [2]. Gingivitis may be defined as "an inflammatory lesion, mediated by host parasite interactions, that is confined to the gingival tissues" [3]. The major cause of gingivitis is an accumulation of microbial plaque in and around the dento-gingival complex, which, when removed, results in complete resolution of the inflammatory lesion [4]. Periodontitis is regarded as "an inflammatory lesion, mediated by complex host-parasite interactions, that leads to the loss of connective tissue attachment to root surface cementum and adjacent alveolar bone" [5]. There are many forms of periodontitis' and the changes associated with periodontitis are irreversible, resulting in tooth loss and substantial morbidity in medically compromised patients, where a focus of infection and subsequent bacteraemia may present a major risk [6]. Substantial data are available in the literature on the role of reactive oxygen species (ROS) and antioxidants in disorders such as the inflammatory lung diseases and in chronic immune mediated conditions such as rheumatoid arthritis [7]. However, remarkably little information is available on the periodontal diseases, which show many of the pathological features of other chronic inflammatory diseases [8]. The periodontal tissues also provide an ideal medium within which to study mechanisms of ROS mediated tissue damage and of antioxidant defense in response to bacterial colonisation, through the non-invasive collection of GCF [9].

Bio-adhesive polymers appear to be particularly attractive for the development of alternative etch free dentin bonding system with an added advantage of additional therapeutic delivery systems to improve intra-dental administration of therapeutic and prophylactic agents if necessary [10]-[15]. Chitosan, which is a biologically safe biopolymer, has been proposed as a bio-adhesive polymer and is of continuous interest to us due to its unique properties and flexibility in a broad range of oral applications reported by others and us recently [16]-[20].

\section{Null Hypothesis}

The objectives of this study are to evaluate the novel chitosan based functional drug delivery systems which can be successfully incorporated into "dual action bioactive restorative materials" containing common antibiotics such as tetracycline, krill oil, aloe and aspirin as commonly used antioxidant species. The novel hydrogels will be investigated with respect to the antioxidant capacity and drug release capacity of the tetracycline from the designer drug delivery system, the use of SEM imaging for the characterization of the surfaces and reactive features of novel materials with antimicrobial potential.

\section{Materials and Methods}

Chitosan (Aldrich, Australia), glycerol (Sigma, USA), glacial acetic acid (E. Merck, Germany) were used as received. The degree of de-acetylation of typical commercial chitosan used in this study is $87 \%$. Chitosan with molecular weight $2.5 \times 10^{3} \mathrm{KD}$ was used in the study. The isoelectric point is $4.0-5.0$. Krill Oil (Aurora Pharmaceuticals, Australia), Aspirin (Safeway, Australia), Ibuprofen (Safeway, Australia) and Naproxen (Safeway, Australia) were used as received. 


\subsection{Preparation of the Gels of the Various Antibiotic Induced Hydrogels}

Chitosan hydrogels have been prepared using the methodology previously described [10]. Briefly, the corresponding antibiotic and antioxidant mixtures, were incorporated by dispersion of corresponding antioxidant powder 0.02 grams in glycerol $(5 \% \mathrm{w} / \mathrm{w})$ using a mortar and a pestle and 1 milliliter of glacial acetic acid (3\% $\mathrm{w} / \mathrm{w})$. The corresponding antioxidant mixtures were incorporated into the mixture and the summary of the newly prepared materials was highlighted in Table 1.

\subsection{Determination of Gel pH}

One gram of the prepared gels was accurately weighed and dispersed in $10 \mathrm{ml}$ of purified water. The $\mathrm{pH}$ of the dispersions was measured using pH meter (HANNA instruments, HI8417, Portugal).

\subsection{In Vitro Tetracycline Release}

The release study was carried out with USP dissolution apparatus type 1, Copley U.K., slightly modified in order to overcome the small volume of the dissolution medium, by using $100 \mathrm{ml}$ beakers instead of the jars. The basket of the dissolution apparatus ( $2.5 \mathrm{~cm}$ in diameter) was filled with $1 \mathrm{gm}$ of tetracycline gel on a filter paper. The basket was immersed to about $1 \mathrm{~cm}$ of its surface in $50 \mathrm{ml}$ of phosphate buffer $\mathrm{pH} 6.8$, at $37^{\circ} \mathrm{C} \pm 0.5^{\circ} \mathrm{C}$ and 100 rpm [21]. Samples (2 ml) were collected at 0.25, 1, 2, 3, 4, 5, 6, 7 and 8, 24, 48, 72, 96, 120, 144, 240 hours [21] and were analyzed spectrophotometrically by U.V. Spectrophotometer (Cintra 5, GBC Scientific equipment, Australia) at The UV-vis absorption spectrum of tetracycline hydrochloride in water is typical of at $361 \mathrm{~nm}$, using the calibration curves $\left(A_{1 \mathrm{~cm}}^{1 \%}=337\right.$ for tetracycline hydrochloride both evaluated in PB, $\mathrm{pH}$ 6.8). Three replicate measurements were performed for each designed formulation [22]. Each sample was replaced by the same volume of phosphate buffer $\mathrm{pH} 6.8$ to maintain its constant volume and sink condition [23].

\subsection{Microbiological Investigations}

A type strain of Staphylococcus aureus (ATCC 12600), obtained from the American Type Culture Collection (Manassas, USA) was used as test bacterium for estimating the antibacterial activity of the hydrogels. The antibacterial activity of the prepared tetracycline/antioxidant chitosan hydrogels were tested using the standard Kirby-Bauer agar disc diffusion method (Bauer, et al.). Five to $6 \mathrm{~mm}$ deep Muller-Hinton agar (Oxoid, Basingstoke, UK) plates were inoculated by streaking a standardized inoculum suspension that match a 0.5 McFarland standard and containing $10^{7}-10^{8}$ colony forming units $/ \mathrm{ml}$ with a throat cotton swab. For each test sample $500 \mu \mathrm{g}$ of hydrogel was applied to a $6 \mathrm{~mm}$ diameter paper disc. The paper discs were placed on the inoculated MullerHinton agar medium and incubated at $37^{\circ} \mathrm{C}$ for 24 hours. The diameter of the zones of growth inhibition was measured with a caliper. Each measurement was done in triplicate and the testing of each sample was repeated 7 times. The antibacterial efficacy of the prepared gels were compared to antibiotic sensitivity discs (Mast Laboratories, Merseyside UL) containing $10 \mu \mathrm{g}$ of tetracycline per disc.

\subsection{Morphology of the Gels}

The samples were prepared by freezing in liquid nitrogen for $10 \mathrm{~min}$, and then were freeze-dried for $24 \mathrm{~h}$. The

Table 1. Gel formulation prepared in the study.

\begin{tabular}{|c|c|c|c|c|c|c|c|}
\hline Gel formulation & & Chitosan (w/w\%) & Tetracycline (w/w\%) & Aspirin (w/w\%) & Aloe (w/w\%) & Krill Oil (w/w\%) & pH \\
\hline Chitosan-H & Gel-1 & 5 & 0 & 0 & 0 & 0 & 5.30 \\
\hline Chitosan-H-TA1 & Gel-2 & 5 & 1 & 0 & 1 & 0 & 6.44 \\
\hline Chitosan-H-TAK2 & Gel-3 & 5 & 1 & 0 & 1 & 1 & 6.54 \\
\hline Chitosan-H-TAs1 & Gel-4 & 5 & 1 & 1 & 0 & 0 & 5.94 \\
\hline Chitosan-H-TAsK2 & Gel-5 & 5 & 1 & 1 & 0 & 1 & 6.34 \\
\hline
\end{tabular}

Where $\mathrm{T}$ is teracycline, $\mathrm{K}$ is Krill oil, As is Asprin, A is Aloe extract. 
prepared samples were fractured in liquid nitrogen using a razor blade. The fractured samples were dried under vacuum, attached to metal stubs, and sputter coated with gold under vacuum for the SEM study. The interior and the surface morphology were observed under scanning electron microscope (SEM, Hitachi S4800, Japan).

\subsection{Gel Stability}

Stability of the gel formulations was also investigated. The organoleptic properties (color, odor), $\mathrm{pH}$, drug content, and release profiles of the gels store at $20^{\circ} \mathrm{C}$ were examined on days $(0,15,30$ and 178$)$. The performance of the hydrogels was not affected by the storage conditions, suggesting remarkable stability of the novel biomaterials under investigations.

\subsection{Studies of Equilibrium Swelling in the Alternative Drug Delivery Systems}

The known weight tea infused-containing dry gels were immersed in $\mathrm{pH} 4.0$, pH 9.0 buffer solutions, respectively, and kept at $25^{\circ} \mathrm{C}$ for $48 \mathrm{~h}$ until equilibrium of swelling had been reached.

The swollen gels were taken out and immediately weighed with microbalance after the excess of water lying on the surfaces was absorbed with a filter paper. The equilibrium swelling ratio (SR) was calculated using the following equation:

$$
S R=\left(W_{s}-W_{d}\right) / W_{d} \times 100 \%
$$

where $W_{s}$ and $W_{d}$ are the weights of the gels at the equilibrium swelling state and at the dry state, respectively [25]. Experiments were repeated in triplicate for each gel specimen and the mean value calculated.

\section{Results}

\subsection{The Characterization of Prepared Chitosan: Tetracycline Containing Gels (Gel-1-Gel-5)}

The SEM images were obtained to characterize the microstructure of the freeze-dried teracycline composite gels and are presented in Figure 1. It could be seen that the gels displayed a homogeneously pore structure. It was thought that the micro-porous structure of the gels could lead to high internal surface areas with low diffusional resistance in the gels. The surfaces of the gels were also presented (Figure 1). The "skin" of the gels can be seen, and the collapse of the surface pores may be due to freeze-drying process.

\subsection{Studies of Equilibrium Swelling in Chitosan Gels (Gel 1, 2, 3, 4 and 5)}

The hydrogels remain in the cylindrical form after swelling. Compared with dry state hydrogels, the swollen state hydrogel volume displays significant increases and are summarized in Figure 2.

Equilibrium swelling ratio (SR) of hydrogels exerts an influence on their release rates. The reduction in equilibrium swelling capacity is due to the formation of a tight network structure in high content. Environmental $\mathrm{pH}$ value has a large effect on the swelling behavior of these gels. From Figure 2, it is clear that the SR value increases with the increase of $\mathrm{pH}$. Such $\mathrm{pH}$ dependent properties of the hydrogels come from the polyelectrolyte nature of chitosan segments in the hydrogel network. Namely, when the $\mathrm{pH}$ value of the buffer solution ( $\mathrm{pH} 9.0$ ) was far higher than the isoelectric point (PI) of GEL (PI 4.0-5.0), the carboxyl groups were de-protonated to carry negative charges, which made molecular chains repulsed to each other. The network became looser and it was easy for the water molecules to diffuse into the cross-linked network. According to above results, we believed that the naproxen results release mechanism could result from the superposition of various effects, such as swelling property of hydrogels, the solubility of the drug and erosion property of matrix; it is not necessarily based on a single factor.

\subsection{In Vitro Release of Antibiotics from Chitosan Gels}

The in vitro release of tetracycline hydrochloride expressed as the cumulative percent of drug released as function of time from the designed formulations of periodontal gels, is presented in Figure 3.

The tetracycline content of the hydrogels was detectable from the UV measurements previously described of 


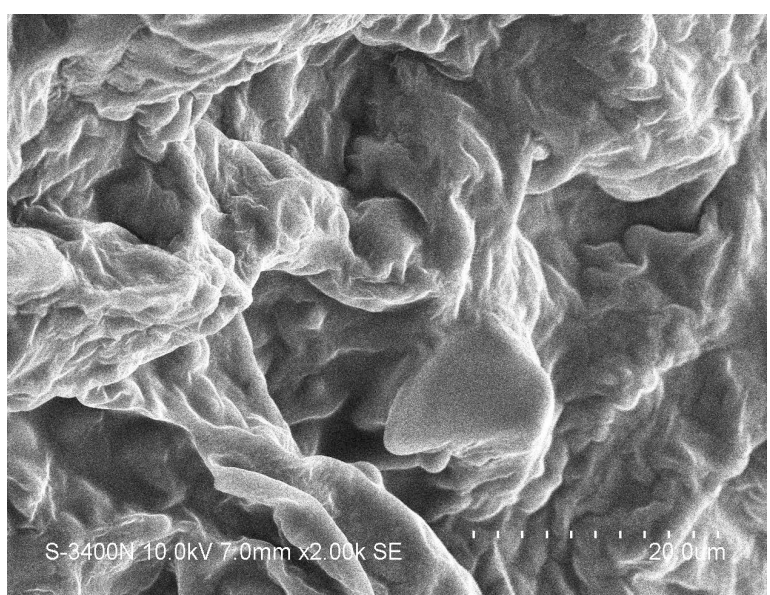

(a)

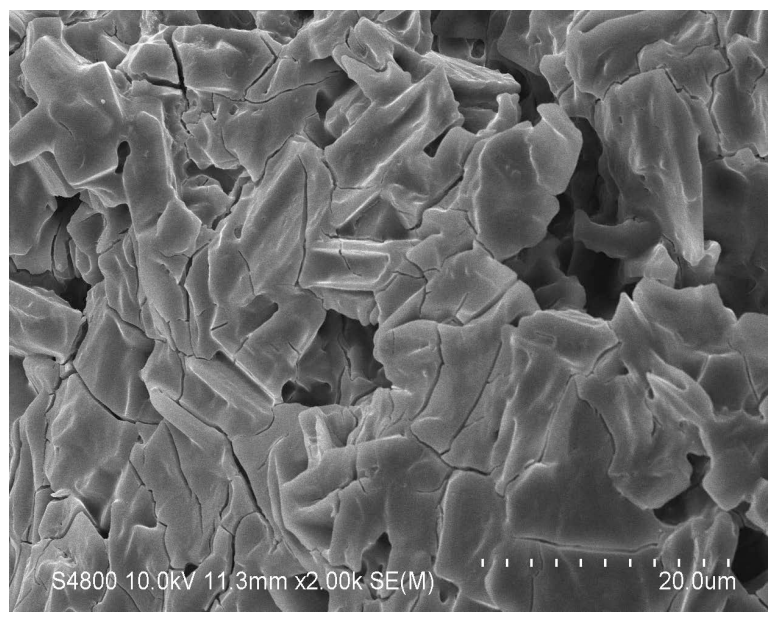

(c)

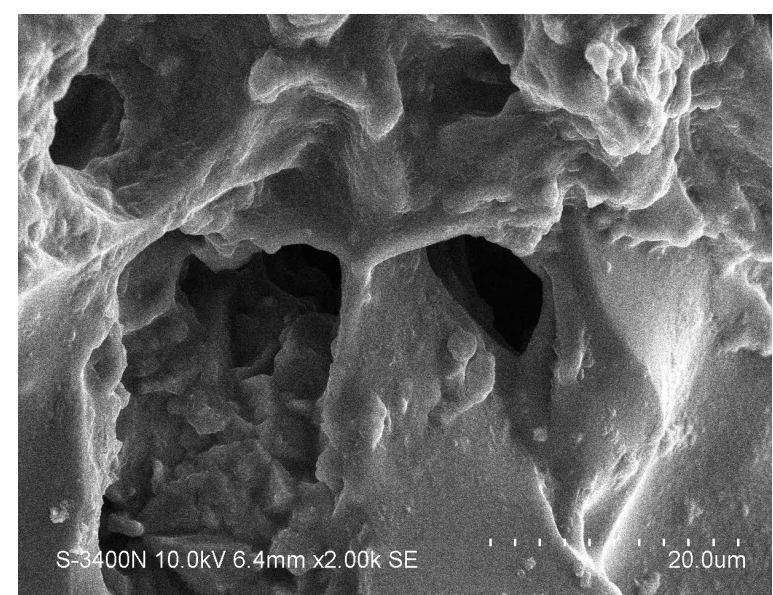

(b)

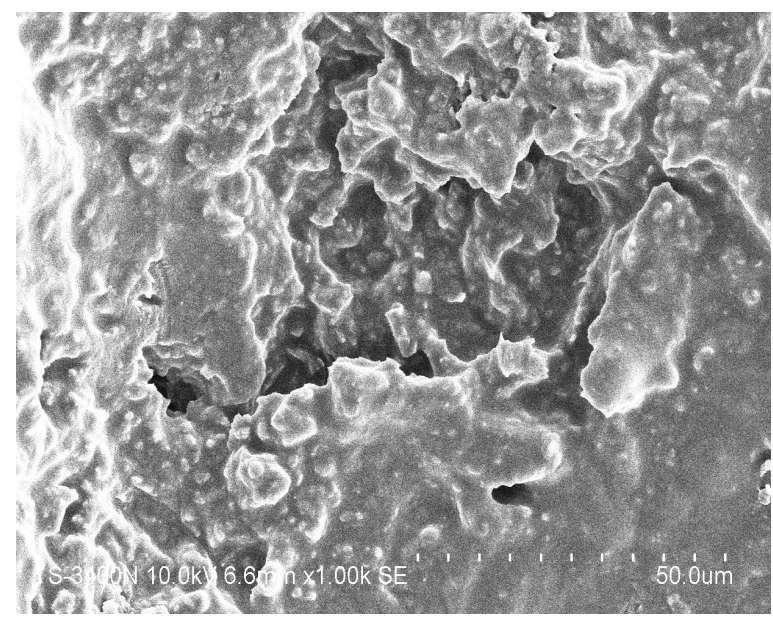

(d)

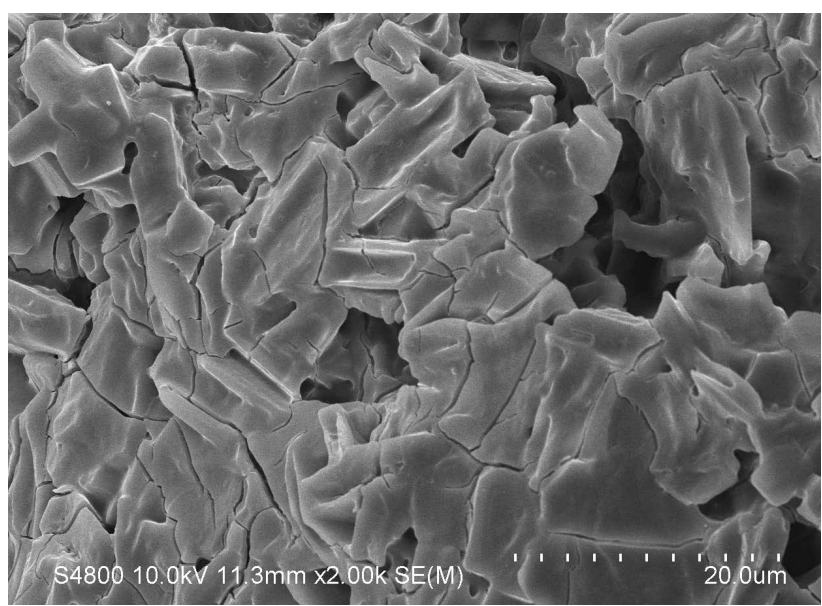

(e)

Figure 1. SEM photographs of interior morphology of the selected gels under investigation for (a) Gel-1, (b) Gel-2, (c) Gel-3, (d) Gel-4, (e) Gel-5.

the hydrogel-containing solution until day 10. Significantly more tetracycline was detected between days 4 and 10 than on days 1 and 3, suggesting the prolonged release of the antibiotic from the hydrogels as demonstrated in Figure 3. 


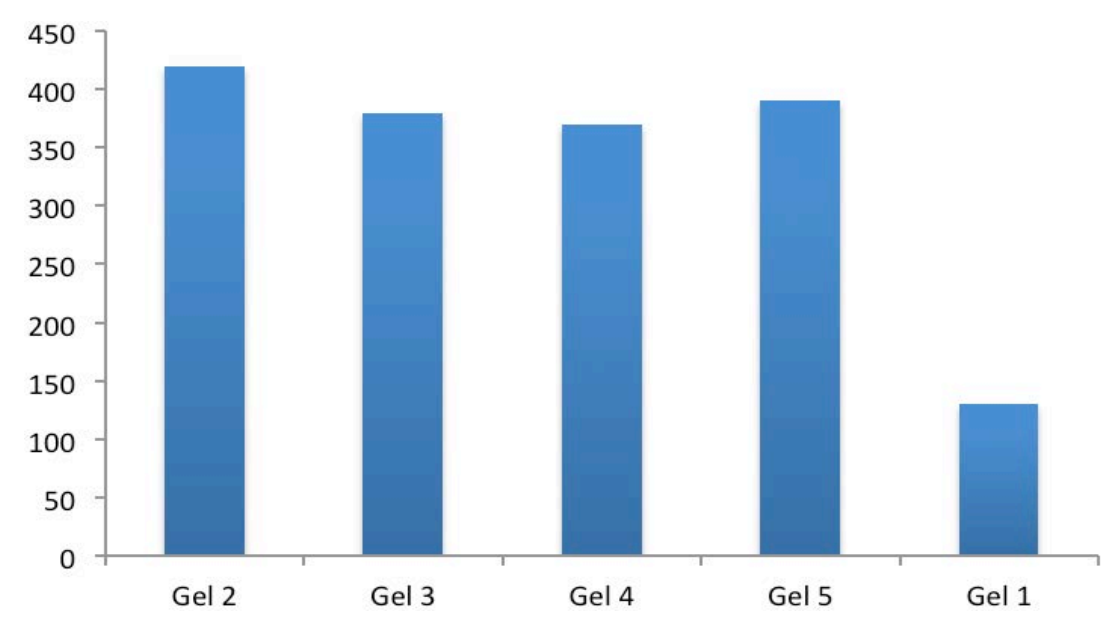

Figure 2. Water uptake degree of the gels Gel-1-Gel-5 $(n=6, p<0.05)$ where $y$-axis is \% of water uptake.

\% cumulative release

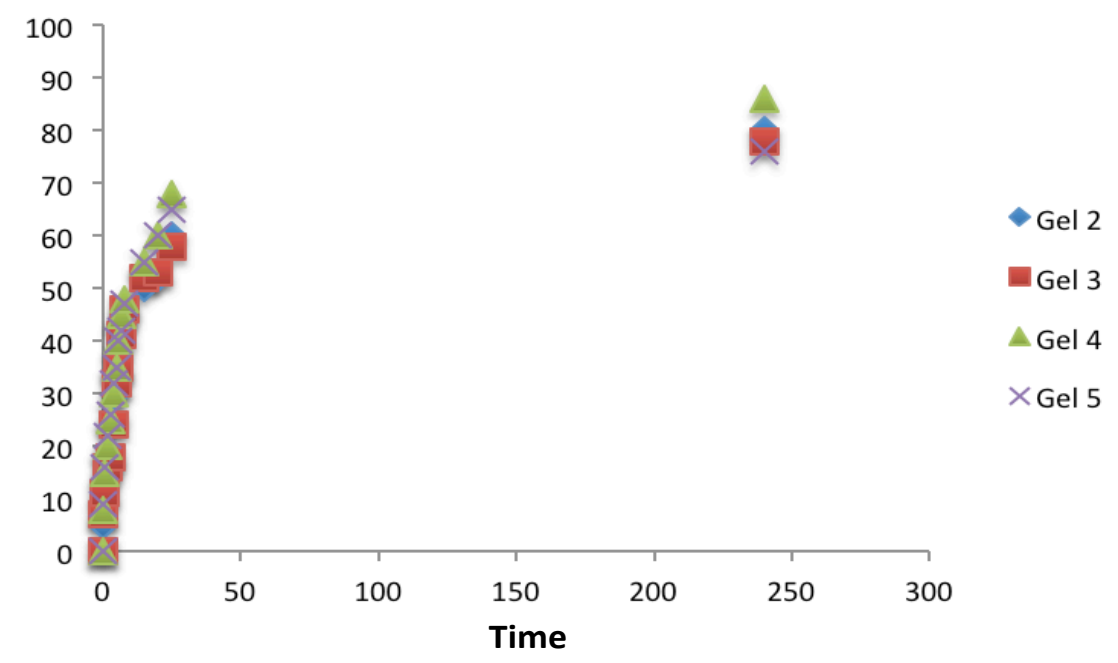

Figure 3. Cumulative release of tetracycline hydrochloride from chitosan gels.

\subsection{In Vitro Microbial Activity of Tetracycline/Antioxidant Containing Chitosan Hydrogels}

Discs with additives but without tetracycline gave no inhibition zones. All the test samples give inhibition zones larger than the clinical breakpoint inhibition zone diameters (European Committee on Antimicrobial Susceptibility Testing, Basel, Switzerland) for S. aureus sensitivity for tetracycline. Using the students T test, no statistically significant difference $(\mathrm{p}<0.05)$ between the averages of the inhibition zone diameters for all the samples except for the hydrogel containing aloe, tetracycline and krill oil were found (Table 2).

\section{Discussion}

\subsection{Antioxidants and Chitosan Looking at the Antioxidant Strength and Extended Stability, Could Macro-Encapsulation Play a Functional Role}

Chitosan, a linear abundant polysaccharide, is selected as the wall material of the delivery system [25]. Due to its biodegradable, biocompatible, muco-adhesive and non-toxic nature, it has been widely used in numerous drug delivery systems. Compared to other delivery systems, chitosan nanoparticles have a special feature. They can adhere to the mucosal surface and transiently open the tight junction between epithelial cells. Some reports 
Table 2. Gel-2, Gel-3, Gel-4 and Gel 5 tested for antibacterial activity against Staphylococcus aureus NCTC 12600.

\begin{tabular}{cccccc}
\hline & \multicolumn{2}{c}{ Tetracycline inhibition zone diameters } & & \\
\hline Sample no. $(\mathrm{n}=10)$ & Tetracycle disc $(10 \mu \mathrm{g})$ & Gel-2 & Gel-3 & Gel-4 & Gel-5 \\
\hline Average & 33.0 & 33.6 & 36.3 & 36.9 & 33.8 \\
Standard deviation & 0.0 & 0.5 & 0.4 & 0.2 & 0.8 \\
\hline
\end{tabular}

Clinical breakpoints for tetracycline: for $30 \mu \mathrm{g} /$ disc $>22 \mathrm{~mm}$ is sensitive and $<19 \mathrm{~mm}$ is resistant.

have indicated that chitosan can increase membrane permeability, both in vitro [24]-[26] and in vivo [27]. Microencapsulation of antioxidants have been important area of research for several years in order to preserve the beneficial effects of antioxidants [28]. In this work, the gelation method was used to prepare the chitosan:aspirin, chitosan:krill oil and chitosan:aspirin:krill Oil microencapsulation. The stability of antioxidant-loaded chitosan complexes has been measured during storage using previously tested established methodology and results suggest there is no significant decomposition observed after 6 months storage at room temperature $\left(24^{\circ} \mathrm{C}\right)$ as antioxidant capacity of the materials stored for 6 month have showed no diminished capacity compare to the freshly prepared hydrogels as indicated in Figures 4 and 5.

\subsection{Investigations into Stability of Antioxidants in the Chitosan Hydrogels during Storage}

Stability of various conventional antioxidants in the newly designed drug delivery system during storage is an important factor to determine whether chitosan-coated nano-size delivery vehicle can protect various conventional antioxidants. So the stability of the microencapsulated antioxidants has been measured by UV absorbance. Stabilities of microencapsulated antioxidants have been compared and after 6 months of storage at $24^{\circ} \mathrm{C}$, the stability of antioxidant-molecular carrier vehicle was not significantly diminished as indicated in Figures 4 and 5. This observation suggests that the antioxidant had been protected by the molecular carrier. Important to note that performance of the antioxidants such as krill oil, aspirin and a 1:1 mixture of krill oil:aspirin was enhanced by the presence of the chitosan, which is a very interesting point in itself as the synergism in increased stability and lower concentration of the active antioxidant with the same or even higher antioxidant capacity can lead to a development of broad range to novel functional drug delivery systems and dual action restorative mate.

\subsection{Insight into Microbiological Investigations}

In the present study, tetracycline was selected because of its wide application, both locally and systemically, in the treatment of periodontal diseases. Tetracycline has been shown to be effective against many of the common periodontopathic bacteria, in particular against Prevotella intermedia and Porphyromonas gingivalis [29]-[31]. In the present study, two commercially available bacterial strains were selected to meet the preliminary goal of whether the activity of antibiotics can be preserved after incorporation into preparations. Our results showed that the cross-linked chitosan sponges were able to deliver active antibiotic for 10 days; however, the effectiveness has yet to be evaluated against potential periodontal pathogens. Because periodontitis is a localized inflammation of the periodontal pocket caused by bacterial infection, current microbiological treatment strategies involve are either the use of systemic antibiotics or a localized delivery system incorporating an antibiotic.

Chitosan hydrogen scaffolds were designed in this study as carriers for antibiotics and showed a steady release of the medication. Three-dimensional chitosan matrices have been shown to be excellent tissue engineering scaffolds for cell attachment and growth. Chitosan has a scalloped structure and has been used in tissue engineering to culture hepatocytes, fibroblasts and cartilage cells because of its ability to promote cell attachment and growth [32]-[38].

Periodontal regeneration with chitosan has been observed after the implantation of chitosan in one-wall alveolar bony defects in dogs [39]. In our investigation, chitosan was selected as the carrier for tetracycline, mainly because it can both carry and deliver the medication, but also because it has other useful bioactivities such as anti-inflammatory properties [40] [41].

\section{Conclusion}

In conclusion, a steady slow release of tetracycline, while maintaining antibiotic effects against the tested bacteria, for at least 10 days was shown from designer chitosan-antioxidant hydrogels. Based on our results, we can 


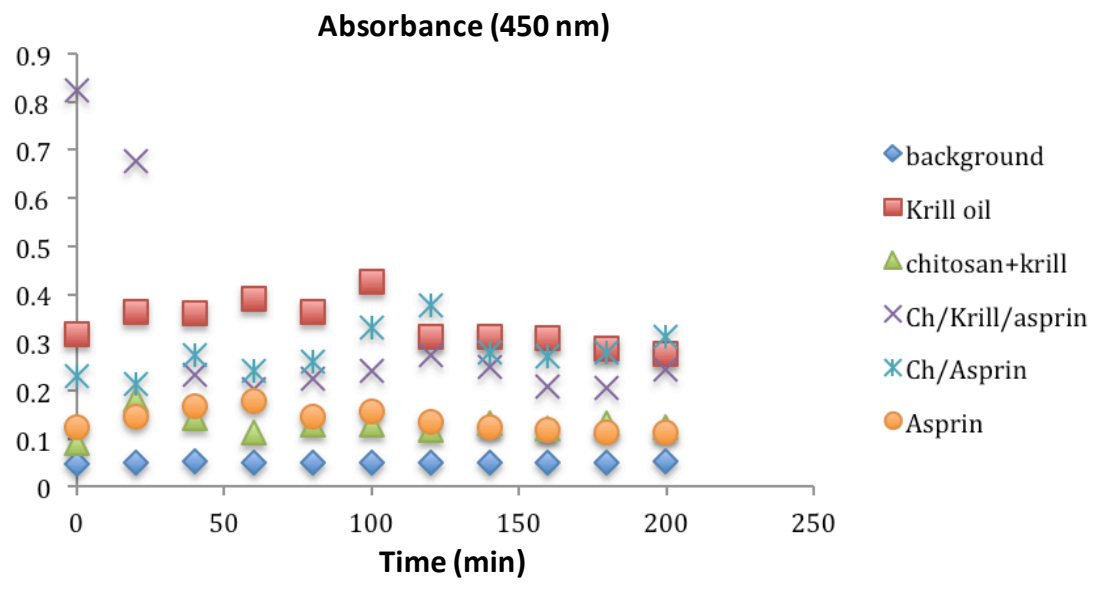

Figure 4. Antioxidant capacity measured at $450 \mathrm{~nm}$ using the previously described spectrophotometric assay to asses the hydrogels and corresponding ingredients antioxidant capacity after 24 hours under storage under ambient temperature condition. Antioxidant capacity was measured during the first 2 hours of exposure.

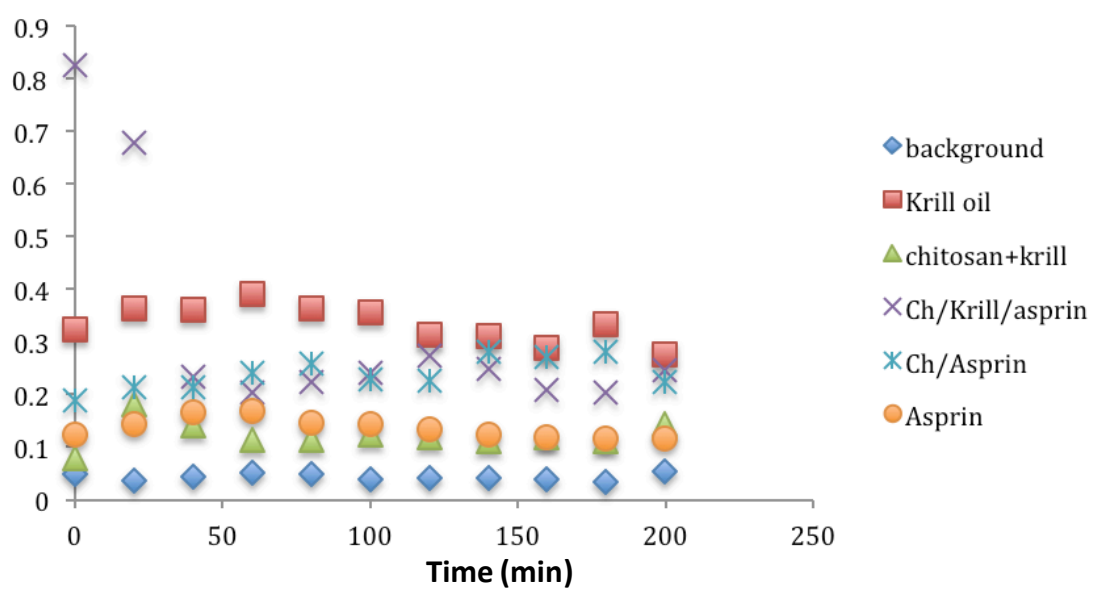

Figure 5. Antioxidant capacity measured at 450nm using the previously described spectrophotometric assay to asses the hydrogels and corresponding ingredients antioxidant capacity after 6 month under storage under ambient temperature condition. Antioxidant capacity was measured during the first 2 hours of exposure.

conclude that the chitosan-antioxidant containing hydrogels are a suitable carrier for tetracycline to be slow-released. Within the limitations of the study design chitosan-antioxidant hydrogels are suitable materials for functional restorative and periodontal applications in vitro. However, future investigations are necessary to validate this hypothesis. The addition of antioxidants to the tetracycline containing prototype delivery system had a beneficial effect on the design of the hydrogel by slowing down the release of tetracycline and thereby enabling a sustainable antifungal activity over time.

\section{References}

[1] Goodson, J.M., Haffajee, A. and Socransky, S.S. (1979) Periodontal Therapy by Local Delivery of Tetracycline. Journal of Clinical Periodontology, 6, 83-92. http://dx.doi.org/10.1111/j.1600-051X.1979.tb02186.x

[2] Goodson, J.M., Cugini, M.A., Kent, R.L., et al. (1991) Multicenter Evaluation of Tetracycline Fiber Therapy: I. Experimental Design, Methods, and Baseline Data. Journal of Periodontal Research, 26, 361-370. http://dx.doi.org/10.1111/j.1600-0765.1991.tb02075.x

[3] Goodson, J.M., Cugini, M.A., Kent, R.L., et al. (1991) Multicenter Evaluation of Tetracycline Fiber Therapy: II. Clinical Response. Journal of Periodontal Research, 26, 371-379. http://dx.doi.org/10.1111/j.1600-0765.1991.tb02076.x 
[4] Newman, M.G., Kornman, K.S. and Doherty, F.M. (1994) A 6-Month Multi-Center Evaluation of Adjunctive Tetracycline Fiber Therapy Used in Conjunction with Scaling and Root Planning in Maintenance Patients: Clinical Results. Journal of Periodontology, 65, 685-691. http://dx.doi.org/10.1902/jop.1994.65.7.685

[5] Soskolne, W.A., Heasman, P.A., Stabholz, A., et al. (1997) Sustained Local Delivery of Chlorhexidine in the Treatment of Periodontitis: A Multi-Center Study. Journal of Periodontology, 68, 32-38. http://dx.doi.org/10.1902/jop.1997.68.1.32

[6] van Steenberghe, D., Bercy, P., Kohl, J., et al. (1993) Subgingival Minocycline Hydrochloride Ointment in Moderate to Severe Chronic Adult Periodontitis: A Randomized, Doubleblind, Vehicle-Controlled, Multicenter Study. Journal of Periodontology, 64, 637-644. http://dx.doi.org/10.1902/jop.1993.64.7.637

[7] Ainamo, J., Lie, T., Ellingsen, B.H., et al. (1992) Clinical Responses to Subgingival Application of a Metronidazole 25\% Gel Compared to the Effect of Subgingival Scaling in Adult Periodontitis. Journal of Clinical Periodontology, 19, 723729. http://dx.doi.org/10.1111/j.1600-051X.1992.tb02535.x

[8] Jeffcoat, M.K., Bray, K.S., Ciancio, S.G., et al. (1998) Adjunctive Use of a Subgingival Controlled Release Chlorhexidine Chip Reduces Probing Depth and Improves Attachment Level Compared with Scaling and Root Planning Alone. Journal of Periodontology, 69, 989-997. http://dx.doi.org/10.1902/jop.1998.69.9.989

[9] Garrett, S., Johnson, L., Drisko, C.H., et al. (1999) Two Multi-Center Studies Evaluating Locally Delivered Doxycycline Hyclate, Placebo Control, Oral Hygiene, and Scaling and Root Planing in the Treatment of Periodontitis. Journal of Periodontology, 70, 490-503. http://dx.doi.org/10.1902/jop.1999.70.5.490

[10] Tonetti, M., Cugini, M.A. and Goodson, J.M. (1990) Zero-Order Delivery with Periodontal Placement of TetracyclineLoaded Ethylene Vinyl Acetate Fibers. Journal of Periodontal Research, 25, 243-249. http://dx.doi.org/10.1111/j.1600-0765.1990.tb00911.x

[11] Greenstein, G. (1987) Effects of Subgingival Irrigation on Periodontal Status. Journal of Periodontology, 58, 827-836. http://dx.doi.org/10.1902/jop.1987.58.12.827

[12] Rethman, M. and Greenstein, G. (1994) Oral Irrigation in the Treatment of Periodontal Diseases. Current Opinion in Periodontology, 99-110.

[13] Khor, E. and Lim, L.Y. (2003) Implantable Applications of Chitin and Chitosan. Biomaterials, 24, 2339-2349. http://dx.doi.org/10.1016/S0142-9612(03)00026-7

[14] Athanasiou, K.A., Shah, A.R., Hernandez, R.J. and LeBaron, R.G. (2001) Basic Science of Articular Cartilage Repair. Clinical Journal of Sport Medicine, 20, 223-247. http://dx.doi.org/10.1016/S0278-5919(05)70304-5

[15] Dornish, M., Kaplan, D. and Skaugrud, O. (2001) Standards and Guidelines for Biopolymers in Tissue-Engineered Medical Products: ASTM Alginate and Chitosan Standard Guides. American Society for Testing and Materials. Annals of the New York Academy of Sciences, 944, 388-397. http://dx.doi.org/10.1111/j.1749-6632.2001.tb03850.x

[16] Hu, Q., Li, B., Wang, M. and Shen, J. (2004) Preparation and Characterization of Biodegradable Chitosan/Hydroxyapatite Nanocomposite Rods via in Situ Hybridization: A Potential Material as Internal Fixation of Bone Fracture. Biomaterials, 25, 779-785. http://dx.doi.org/10.1016/S0142-9612(03)00582-9

[17] Risbud, M.V. and Bhonde, R.R. (2000) Polyacrylamidechitosan Hydrogels: In Vitro Biocompatibility and Sustained Antibiotic Release Studies. Drug Delivery, 7, 69-75. http://dx.doi.org/10.1080/107175400266623

[18] Chow, K.S. and Khor, E. (2000) Novel Fabrication of Open-Pore Chitin Matrixes. Biomacromolecules, 1, 61-67. http://dx.doi.org/10.1021/bm005503b

[19] Senel, S., Ikinci, G., Kas, S., Yousefi-Rad, A., Sargon, M.F. and Hincal, A.A. (2000) Chitosan Films and Hydrogels of Chlorhexidine Gluconate for Oral Mucosal Delivery. International Journal of Pharmaceutics, 193, 197-203. http://dx.doi.org/10.1016/S0378-5173(99)00334-8

[20] Needleman, I.G., Smales, F.C. and Martin, G.P. (1997) An Investigation of Bioadhesion for Periodontal and Oral Mucosal Drug Delivery. Journal of Clinical Periodontology, 24, 394-400. http://dx.doi.org/10.1111/j.1600-051X.1997.tb00203.x

[21] Lin, D.M., Kalachandra, S., Valiyaparambil, J. and Offenbacher, S. (2003) A Polymeric Device for Delivery of AntiMicrobial and Anti-Fungal Drugs in the Oral Environment: Effect of Temperature and Medium on the Rate of Drug Release. Dental Materials, 19, 589-596. http://dx.doi.org/10.1016/S0109-5641(02)00109-4

[22] Mi, F.L., Shyu, S.S., Chen, C.T. and Schoung, J.Y. (1999) Porous Chitosan Microsphere for Controlling the Antigen Release of Newcastle Disease Vaccine: Preparation of Antigen Adsorbed Microsphere and in Vitro Release. Biomaterials, 20, 1603-1612. http://dx.doi.org/10.1016/S0142-9612(99)00064-2

[23] Park, Y.J., Lee, J.Y., Yeom, H.R., et al. (2005) Injectable Polysaccharide Microcapsules for Prolonged Release of Minocycline for the Treatment of Periodontitis. Biotechnology Letters, 27, 1761-1766.

http://dx.doi.org/10.1007/s10529-005-3550-7 
[24] Chung, T.W., Lu, Y.F., Wang, S.S., Lin, Y.S. and Chu, S.H. (2002) Growth of Human Endothelial Cells on Photochemically Grafted Gly-Arg-Gly-Asp (GRGD) Chitosans. Biomaterials, 23, 4803-4809. http://dx.doi.org/10.1016/S0142-9612(02)00231-4

[25] Flemmig, T.F., Weinacht, S., Rudiger, S., Rumetsch, M., Jung, A. and Klaiber, B. (1996) Adjunctive Controlled Topical Application of Tetracycline $\mathrm{HCl}$ in the Treatment of Localized Persistent or Recurrent Periodontitis. Effects on Clinical Parameters and Elastasealpha1-Proteinase Inhibitor in Gingival Crevicular Fluid. Journal of Clinical Periodontology, 23, 914-921. http://dx.doi.org/10.1111/j.1600-051X.1996.tb00511.x

[26] Odim, J., Laks, H., Allada, V., Child, J., Wilson, S. and Gjertson, D. (2005) Results of Aortic Valvesparing and Restoration with Autologous Pericardial Leaflet Extensions in Congenital Heart Disease. Annals of Thoracic Surgery, 80, 647-653. http://dx.doi.org/10.1016/j.athoracsur.2005.03.060

[27] Myken, P.S. (2005) Seventeen-Year Experience with the St Jude Medical Biocor Porcine Bioprosthesis. Journal of Heart Valve Disease, 14, 486-492.

[28] Lu, H.K., Lee, S.Y. and Lin, F.P. (1998) Elastic Modulus, Permeation Time and Swelling Ratio of a New Porcine Dermal Collagen Membrane. Journal of Periodontal Research, 33, 243-248. http://dx.doi.org/10.1111/j.1600-0765.1998.tb02196.x

[29] Ishihara, M., Ono, K., Sato, M., et al. (2001) Acceleration of Wound Contraction and Healing with a Photocrosslinkable Chitosan Hydrogel. Wound Repair and Regeneration, 9, 513-521. http://dx.doi.org/10.1046/j.1524-475x.2001.00513.x

[30] Guo, J.F., Jourdian, G.W. and MacCallum, D.K. (1989) Culture and Growth Characteristics of Chondrocytes Encapsulated in Alginate Beads. Connective Tissue Research, 19, 277-297. http://dx.doi.org/10.3109/03008208909043901

[31] Kawase, M., Michibayashi, N., Nakashima, Y., Kurikawa, N., Yagi, K. and Mizoguchi, T. (1997) Application of Glutaraldehyde-Crosslinked Chitosan as a Scaffold for Hepatocyte Attachment. Biological \& Pharmaceutical Bulletin, 20, 708-710. http://dx.doi.org/10.1248/bpb.20.708

[32] Koyano, T., Minoura, N., Nagura, M. and Kobayashi, K. (1998) Attachment and Growth of Cultured Fibroblast Cells on PVA/Chitosan Blended Hydrogels. Journal of Biomedical Materials Research, 39, 486-490. http://dx.doi.org/10.1002/(SICI)1097-4636(19980305)39:3<486::AID-JBM20>3.0.CO;2-7

[33] Lee, Y.M., Park, Y.J., Lee, S.J., et al. (2000) Tissue Engineered Bone Formation Using Chitosan/Tricalcium Phosphate Sponges. Journal of Periodontology, 71, 410-417. http://dx.doi.org/10.1902/jop.2000.71.3.410

[34] Zhang, Y. and Zhang, M. (2002) Three-Dimensional Macroporous Calcium Phosphate Bioceramics with Nested Chitosan Sponges for Load-Bearing Bone Implants. Journal of Biomedical Materials Research, 61, 1-8. http://dx.doi.org/10.1002/jbm.10176

[35] Zhao, F., Yin, Y., Lu, W.W., et al. (2002) Preparation and Histological Evaluation of Biomimetic Three-Dimensional Hydroxyapatite/Chitosan-Gelatin Network Composite Scaffolds. Biomaterials, 23, 3227-3234. http://dx.doi.org/10.1016/S0142-9612(02)00077-7

[36] Anitua, E. (1999) Plasma Rich in Growth Factors: Preliminary Results of Use in the Preparation of Future Sites for Implants. International Journal of Oral \& Maxillofacial Implants, 14, 529-535.

[37] Klokkevold, P.R., Vandemark, L., Kenney, E.B. and Bernard, G.W. (1996) Osteogenesis Enhanced by Chitosan (Poly-N-Acetyl Glucosaminoglycan) in Vitro. Journal of Periodontology, 67, 1170-1175. http://dx.doi.org/10.1902/jop.1996.67.11.1170

[38] Mizuno, K., Yamamura, K., Yano, K., et al. (2003) Effect of Chitosan Film Containing Basic Fibroblast Growth Factor on Wound Healing in Genetically Diabetic Mice. Journal of Biomedical Materials Research, 64A, 177-181. http://dx.doi.org/10.1002/jbm.a.10396

[39] Park, J.S., Choi, S.H., Moon, I.S., Cho, K.S., Chai, J.K. and Kim, C.K. (200) Eight-Week Histological Analysis on the Effect of Chitosan on Surgically Created One-Wall Intrabony Defects in Beagle Dogs. Journal of Clinical Periodontology, 30, 443-453. http://dx.doi.org/10.1034/j.1600-051X.2003.10283.x

[40] Chou, T.C., Fu, E. and Shen, E.C. (2003) Chitosan Inhibits Prostaglandin E2 Formation and Cyclooxygenase-2 Induction in Lipopolysaccharide-Treated RAW 264.7 Macrophages. Biochemical and Biophysical Research Communications, 308, 403-407. http://dx.doi.org/10.1016/S0006-291X(03)01407-4

[41] Bauer, A.W., Kirby, W.M.M., Sherris, J.C. and Turck, M. (1966) Antibiotic Susceptibility Testing by a Standardized Single Disc Method. American Journal of Clinical Pathology, 36, 493-496. 